\title{
Recomendações para a prática do treinamento de resistência muscular em idosos durante a pandemia do COVID-19
}

Recommendations on the practice of resistance training for older people during the COVID-19 pandemic

Recomendaciones para el entrenamiento de resistencia muscular en ancianos durante la pandemia del COVID-19

\author{
Carlos José Nogueira \\ Universidade Federal do Estado do Rio de Janeiro (UNIRIO), Brasil \\ Escola Preparatória de Cadetes do Ar (EPCAR). \\ Força Aérea Brasileira (FAB), Brasil \\ carlosjn29@yahoo.com.br
}

(D) https://orcid.org/0000-0003-2136-2177

\section{Alisson Gomes da Silva}

Universidade Federal de Viçosa (UFV), Brasil Escola

Preparatória de Cadetes do Ar (EPCAR).

Força Aérea Brasileira (FAB), Brasil

alisson.gomes@ufv.br

(iD https://orcid.org/0000-0002-2086-3431

Samuel Gonçalves Almeida da Encarnação

Universidade Federal de Viçosa (UFV), Brasil

samuel.encarnacao@ufv.br

(D) https://orcid.org/0000-0003-2965-2777

\section{Délson Lustosa Figueirêdo}

Universidade Federal do Estado do Rio de Janeiro

(UNIRIO), Brasil

delsonfig@hotmail.com

(D) https://orcid.org/0000-0002-4537-1775

\section{Estélio Henrique Martin Dantas}

Universidade Federal do Estado do Rio de Janeiro

(UNIRIO), Brasil

Universidade Tiradentes de Aracajú (UNIT), Brasil

estelio.dantas@unirio.br

(D) https://orcid.org/0000-0003-0981-8020

\section{Resumo:}

O treinamento de resistência muscular (TR) constitui uma modalidade alternativa de exercício para promover e preservar a saúde física e independência dos idosos durante a pandemia do COVID-19. O objetivo desta revisão integrativa foi analisar a produção científica em relação as recomendaçôes do TR para melhoria da função muscular e desempenho funcional em idosos em face da COVID-19. Foi realizada uma busca sensibilizada nas bases de dados MEDLINE / PubMed, Embase e Web of Science, sem restrição de data, nos idiomas inglês, espanhol e português, com os descritores: infecção por coronavírus (coronavirus infections) e

\section{Recepción: 10 Septiembre de 2021 | Aprobación: 13 Septiembre 2021 | Publicación: 01 Octubre 2021}

Cita sugerida: Nogueira, C. J., Silva, A. G. da., Encarnação, S. G. A. da., Figueirêdo, D. L. y Dantas, E. H. M. (2021). Recomendações para a prática do treinamento de resistência muscular em idosos durante a pandemia do COVID-19. Educación Física y Ciencia, 23(4), e195. https://doi.org/10.24215/23142561e195 
treinamento de resistência (resistance training). Foram recuperados inicialmente 139 artigos; 13 artigos foram pré-selecionados e 7 elegíveis quanto a pergunta de pesquisa. As evidências apresentadas sugerem que a prática do TR, domiciliar, por meio de exercícios de fortalecimento com o peso do próprio corpo, constitui uma estratégia de intervenção eficiente que impacta positivamente na qualidade de vida dos idosos.

PalaVras-Chave: Exercício físico, Treinamento de resistência muscular, Pandemia do covid-19, Idoso.

\section{Abstract:}

Resistance training (RT) is an alternative exercise modality that can promote and preserve the physical health and independence of older people during the COVID-19 pandemic. The purpose of this integrative review was to analyze the scientific production related to RT recommendations aiming at enhancing the functional performance and muscle function in older people during the COVID-19 pandemic. Sensitive searches were conducted in MEDLINE / PubMed, Embase, and Web of Science using the descriptors "coronavirus infection" and "resistance training". Articles published in English, Spanish, or Portuguese were considered without date restriction. Results: In the initial search, 139 articles were retrieved; 13 were pre-selected and 7 were eligible considering the research question. The evidence presented suggests that home RT through bodyweight-based exercises represents an effective intervention strategy that positively impacts on the quality of life in older people.

KEYWORDS: Exercises, Resistance training, COVID-19 pandemic, Aged people.

\section{ReSUMEN:}

El entrenamiento de resistencia muscular (TR) constituye una modalidad alternativa de ejercício para promover y preservar la salud física e independencia de los ancianos durante la pandemia de covid-19. El objetivo de esta revisión integradora fue de analizar la producción científica en relación a las recomendaciones de la TR para mejora de la función muscular y desempeño funcional en ancianos frente al COVID-19. Fue realizada una búsqueda sensible en las bases de datos MEDLINE/PubMed, Embase y Web of Science, sin restricción de fecha, en los idiomas: inglés, español y portugués; con los descriptores: infección por coronavirus (coronavirus infections) y entrenamiento de resistencia (resistance training). Fueron recuperados inicialmente 139 artículos: 13 artículos fueron preseleccionados y 7 elegibles en relación a la pregunta de investigación. Las evidencias presentadas sugieren que la práctica de TR, domiciliar, por medio de ejercícios de fortalecimiento con el peso del próprio cuerpo, constituye una estratégia de intervención eficiente, que impacta positivamente en la calidad de vida de los ancianos.

Palabras clave: Ejercício físico, entrenamiento de resistencia muscular, Pandemia de covid-19, ancianos.

\section{INTRODUÇÃO}

O surto generalizado de coronavírus (COVID-19) no mundo levantou preocupações sobre a viabilidade da prática de atividades físicas e exercícios (Shirvani \& Rostamkhani, 2020). A atual pandemia do COVID-19 apresenta um inesperado desafio à saúde pública. Neste cenário, o exercício físico é visto como o principal aliado na prevenção e proteção do organismo contra esse vírus (Nogueira, Cortez, Leal, \& Dantas, 2021).

Os idosos são mais suscetíveis a infecções virais devido ao seu sistema imune normalmente deprimido pela idade avançada (imunossenescência), sendo a população mais afetada por essa pandemia (Moro \& Paoli, 2020). Dessa forma, a manutenção do condicionamento físico durante o distanciamento social é fundamental para promover a saúde e prevenir futuras condições crônicas decorrentes do sedentarismo (Scartoni et al., 2020).

Segundo Gentil et al. (2020) o treinamento de resistência muscular (TR) pode ser uma ferramenta eficaz para neutralizar as consequências adversas oriundas da redução da mobilidade imposta pelo distanciamento, como a atrofia muscular, perda progressiva da força muscular e reduções nas capacidades neuromusculares e mecânicas, que levam a rápida perda da independência em idosos.

Embora o Colégio Americano de Medicina do Esporte tenha divulgado recentemente um guia com as principais recomendações sobre a atividade física durante a pandemia do coronavírus, sugerindo duas sessões por semana de treinamento de força muscular (American College of Sports Medicine, 2020), as diretrizes recentes sobre a prescrição de exercícios para combater os efeitos deletérios do COVID-19 não abordam adequadamente o treinamento de resistência muscular como estratégia de exercícios domiciliares (Scartoni et al. 2020). 
Portanto, o objetivo desta revisão é avaliar e sintetizar as evidências científicas em relação as recomendações do treinamento de resistência muscular para melhoria da função muscular e desempenho funcional em idosos em face da COVID-19.

\section{Metodologia}

Trata-se de uma revisão integrativa da literatura, modalidade que consiste em resumir e analisar resultados de pesquisas, além divulgar sínteses de conhecimentos científicos produzidos acerca de um fenômeno de interesse (Oliveira, Oliveira-Cardoso, Silva, \& Santos 2020).

O estudo cumpriu criteriosamente as seguintes etapas: formulação da questão norteadora; seleção dos estudos, extração dos dados dos estudos incluídos, avaliação e interpretação dos resultados e, por fim, apresentação da revisão do conhecimento produzido (Beyea, 1998).

A questão de pesquisa e as estratégias de busca utilizadas foram baseadas no modelo População, Intervenção, Comparação, Resultado (PICO) (Santos, Pimenta, \& Nobre, 2007).

Com base no exposto, idosos saudáveis ( $\geq 60$ anos), independentemente do sexo, foram utilizados como "População"; para "Intervenção", foram considerados estudos sobre o treinamento de resistência muscular; para "Controle", foi adotado o critério "não aplicável"; e como "Resultado", foi considerado o desfecho primário sobre a melhoria da função muscular e desempenho funcional durante a pandemia do COVID-19. Assim, ficou estabelecida a seguinte pergunta PICO: Quais as principais recomendações com relação ao treinamento de resistência muscular sobre a melhoria da função muscular e desempenho funcional em idosos durante a pandemia do COVID-19?

\section{Processo de Seleção dos estudos, estratégia de busca e extração dos dados}

Uma busca sistemática foi realizada nas bases de dados eletrônicas MEDLINE via PubMed, Embase e Web of Science, sem restrição de data, até 30 de abril de 2021. Foram utilizados os termos selecionados nos Descritores em Ciências da Saúde (DeCS) e do Medical Subjetc Headings (MeSH): infecção por coronavírus (coronavirus infections) e treinamento de resistência (resistance training). Os descritores e seus sinônimos foram combinados e cruzados com os operadores booleanos "OR" e "AND", respectivamente. A seguinte estratégia de busca foi utilizada na MEDLINE / PubMed e adaptada as demais bases de dados: ("Coronavirus Infections"[Mesh] OR "COVID-19" OR "Coronavirus Infection" OR "Infection, Coronavirus" OR "Infections, Coronavirus" OR "Middle East Respiratory Syndrome" OR "Novel Coronavirus Pneumonia" OR "SARS-CoV-2" OR "2019 novel coronavirus Pneumonia" OR "2019-novel coronavirus Pneumonia" OR "2019 novel coronavirus Pandemic" OR "2019-nCoV Pandemic" OR "2019-nCoV Pneumonia" OR "MERS (Middle East Respiratory Syndrome)") AND ("Resistance Training"[Mesh] OR "Strength Training" OR "Training, Resistance" OR "Training, Strength").

Foram incluídos somente estudos nos idiomas inglês, espanhol e português, que responderam à questão norteadora. As evidências compreenderam artigos originais randomizados e não randomizados, revisões e editoriais.

O processo de seleção dos estudos foi realizado por dois revisores independentes, que avaliaram os títulos e resumos das referências recuperadas nas bases de dados, por meio da plataforma Rayyan (https://rayyan.qcri .org) (Ouzzani, Hammady, Fedorowicz, \& Elmagarmid 2016). Dúvidas ou inconsistências foram discutidas posteriormente, até que se estabelecessem os consensos. $\mathrm{Na}$ sequência, foi realizada a avaliação do texto na íntegra dos estudos pré-selecionados para confirmação da elegibilidade.

A extração dos dados ocorreu por meio de um formulário padronizado. Os revisores, de forma independente, conduziram a extração de dados com relação às características metodológicas dos estudos, 
intervenções e resultados. As diferenças foram resolvidas por consenso. Os seguintes dados foram analisados de forma descritiva e independente por dois pesquisadores: autores e ano de publicação, tipo de estudo, objetivo e evidências.

\section{Resultados}

Foram recuperados 139 artigos na busca inicial. $\mathrm{Na}$ sequência, 126 artigos foram rejeitados pelo título, resumo e duplicados, restando 13 artigos pré-selecionados para a leitura na íntegra. Após esta etapa, foram excluídos 6 artigos por apresentarem diferentes intervenções e populações. Ao final do processo de seleção restaram 7 artigos que preencheram os critérios de inclusão.

O processo de seleção dos estudos seguiu as diretrizes dos itens de relatórios preferenciais para revisões sistemáticas e meta-análises PRISMA Statement (Page et al., 2021), conforme apresentado na figura 1. Foi realizada uma síntese qualitativa dos estudos selecionados, apresentando as principais evidências dos estudos elegíveis (Tabela 1 ).

FIGURA 1

Fluxograma da seleção dos artigos (Prisma Flow)

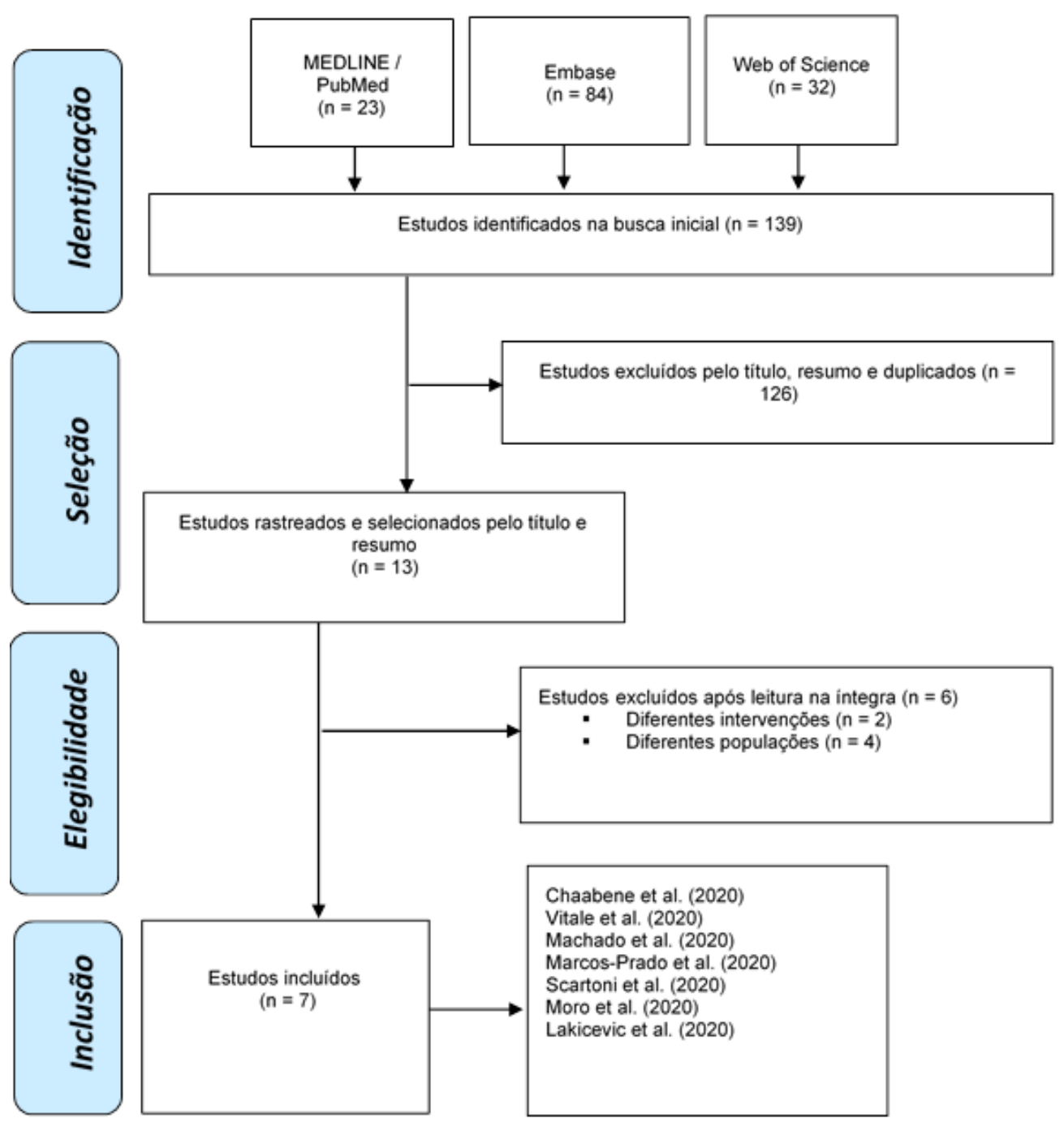

Fonte: Autor, 2020 adaptação de Page et al. (2020). 
TABELA 1

Sumarização das principais evidências encontradas.

\begin{tabular}{|c|c|c|c|}
\hline Autor & $\begin{array}{l}\text { Tipo de } \\
\text { Estudo }\end{array}$ & Objetivo & Evidência \\
\hline $\begin{array}{c}\text { Chaabene } \\
\text { et al. } \\
(2020)\end{array}$ & $\begin{array}{c}\text { Revisão } \\
\text { Sistemática } \\
\text { com Meta- } \\
\text { análise }\end{array}$ & $\begin{array}{l}\text { Investigar os efeitos de } \\
\text { programas de exercícios fisicos } \\
\text { domiciliares (TR individual ou } \\
\text { combinado) sobre medidas de } \\
\text { aptidão fisica em idosos } \\
\text { saudáveis. }\end{array}$ & $\begin{array}{l}\text { Dezessete ensaios clínicos randomizados foram } \\
\text { incluídos com (n=1.477 participantes). Os } \\
\text { resultados indicaram pequenos efeitos do } \\
\text { treinamento doméstico na força muscular (DMP= } \\
0,30 \text { ), potência muscular (DMP }=0,43 \text { ), } \\
\text { resistência muscular (DMP= } 0,28 \text { ) e equilibrio } \\
(\mathrm{DMP}=0,28 \text { ). Não foram encontrados efeitos } \\
\text { significativos do TR individual em comparação } \\
\text { ao TR combinado sobre a força muscular e } \\
\text { equilibrio. O TR individual promoveu efeitos } \\
\text { moderados na força muscular e equilibrio. Os } \\
\text { resultados indicaram efeitos maiores para > } 3 \\
\text { sessões semanais e } \leq 30 \text { min por sessão na força } \\
\text { muscular e equilibrio em idosos saudáveis, } \\
\text { independentemente do tipo de treinamento. }\end{array}$ \\
\hline $\begin{array}{l}\text { Vitale et } \\
\text { al. }(2020)\end{array}$ & $\begin{array}{c}\text { Estudo } \\
\text { randomizado } \\
\text { controlado }\end{array}$ & $\begin{array}{l}\text { Avaliar o efeito de um } \\
\text { programa de TR doméstico de } \\
\text { seis meses sobre a saúde } \\
\text { muscular e o desempenho físico } \\
\text { em idosos saudáveis durante a } \\
\text { condição única de } \\
\text { confinamento doméstico } \\
\text { causada pela pandemia de } \\
\text { COVID-19 }\end{array}$ & $\begin{array}{l}\text { Os idosos foram randomizados em grupo } \\
\text { experimental (TR domiciliar durante } 6 \text { meses; } n \\
=5 \text { ) e controle (sem treinamento; } n=4 \text { ). Os } \\
\text { idosos do grupo experimental melhoraram a força } \\
\text { dos membros inferiores durante o periodo de } \\
\text { isolamento, e esses beneficios foram } \\
\text { provavelmente associados ao programa de } \\
\text { treinamento de resistência baseado em casa. }\end{array}$ \\
\hline $\begin{array}{c}\text { Machado } \\
\text { et al. } \\
(2020)\end{array}$ & $\begin{array}{l}\text { Artigo de } \\
\text { Opinião }\end{array}$ & $\begin{array}{l}\text { Evidenciar a importância do TR } \\
\text { domiciliar como uma estratégia } \\
\text { para mitigar a inatividade fisica } \\
\text { e melhorar ou manter a função } \\
\text { muscular e o desempenho } \\
\text { funcional em idosos. }\end{array}$ & $\begin{array}{l}\text { Como consequência negativa do confinamento } \\
\text { domiciliar, observou-se aumento do tempo de } \\
\text { permanência em casa e maior comportamento } \\
\text { sedentário. Portanto, são necessárias estratégias } \\
\text { que ajudem a evitar a inatividade física e } \\
\text { promovam a saúde fisica dos idosos. Nesse } \\
\text { cenário, o TR doméstico deve ser incentivado por } \\
\text { ser uma modalidade alternativa de exercício para } \\
\text { promover e manter a saúde fisica neste periodo. }\end{array}$ \\
\hline
\end{tabular}




\begin{tabular}{|c|c|c|c|}
\hline $\begin{array}{l}\text { Marcos- } \\
\text { Pardo et } \\
\text { al. (2020) }\end{array}$ & $\begin{array}{l}\text { Artigo de } \\
\text { Opinião }\end{array}$ & $\begin{array}{l}\text { Apresentar o TR domiciliar } \\
\text { como proposta relevante para } \\
\text { manter a saúde } \\
\text { musculoesquelética } \\
\text { psicossocial em adultos mais } \\
\text { velhos durante a pandemia do } \\
\text { COVID-19. }\end{array}$ & $\begin{array}{l}\text { O TR domiciliar envolvendo a resistência } \\
\text { elástica, como uma maneira simples de fornecer } \\
\text { sobrecarga progressiva, demonstrou oferecer } \\
\text { beneficios semelhantes aos equipamentos de TR } \\
\text { tradicionais normalmente encontrados em } \\
\text { ginásios. }\end{array}$ \\
\hline $\begin{array}{l}\text { Scartoni } \\
\text { et al. } \\
(2020)\end{array}$ & $\begin{array}{l}\text { Mini } \\
\text { Revisão }\end{array}$ & $\begin{array}{l}\text { Discutir as implicações e } \\
\text { importância do exercício fisico } \\
\text { e sistema imunológico em } \\
\text { idosos durante a pandemia do } \\
\text { COVID-19. }\end{array}$ & $\begin{array}{l}\text { Recomenda-se o TR sem carga e treinamento } \\
\text { intervalado de alta intensidade como possiveis } \\
\text { estratégias de exercícios domiciliares durante a } \\
\text { pandemia do COVID-19. No entanto, é } \\
\text { necessário monitorar a intensidade } \\
\text { principalmente durante e após as sessões de } \\
\text { treinamento. }\end{array}$ \\
\hline $\begin{array}{l}\text { Moro e } \\
\text { Paoli } \\
(2020)\end{array}$ & $\begin{array}{ll}\text { Artigo de } \\
\text { Opinião }\end{array}$ & $\begin{array}{l}\text { Recomendar o treinamento de } \\
\text { resistência e nutrição adequada } \\
\text { para superar a perda muscular } \\
\text { devido ao sedentarismo durante } \\
\text { a pandemia do COVID-19. }\end{array}$ & $\begin{array}{l}\text { Em função das restrições impostas pelo } \\
\text { isolamento social, é imprescindivel manter os } \\
\text { exercícios, mesmo com baixa carga e sessões } \\
\text { esporádicas de exercícios, durante os períodos de } \\
\text { inatividade. Uma vez retornados aos níveis usuais } \\
\text { de atividade fisica, a combinação de exercícios } \\
\text { resistidos e estímulos nutricionais (balanço } \\
\text { energético adequado e ingestão de proteínas de } \\
\text { alta qualidade) parece ser a estratégia mais eficaz } \\
\text { para contrabalançar as perdas no músculo } \\
\text { esquelético devido ao desuso. }\end{array}$ \\
\hline $\begin{array}{l}\text { Lakicevic } \\
\text { et al. } \\
(2020)\end{array}$ & $\begin{array}{ll}\text { Artigo de } \\
\text { Opinião }\end{array}$ & $\begin{array}{l}\text { Orientar quanto a prescrição de } \\
\text { TR para idosos durante a } \\
\text { pandemia de COVID-19. }\end{array}$ & $\begin{array}{l}\text { Sugerem à população idosa durante a pandemia a } \\
\text { prática do TR domiciliar pelo menos duas vezes } \\
\text { por semana em dias não consecutivos. O TR deve } \\
\text { ser planejado para incluir } 8 \text { a } 10 \text { exercícios } \\
\text { (envolvendo os principais grupos musculares), } \\
\text { permitindo } 1 \text { a } 3 \text { séries por exercício enquanto } \\
\text { realiza } 10 \text { a } 15 \text { repetições por série. Deve-se } \\
\text { priorizar os exercícios realizados com o próprio } \\
\text { peso corporal, pois são seguros e eficazes quando } \\
\text { executados de maneira correta. }\end{array}$ \\
\hline
\end{tabular}

$\mathrm{TR}=$ Treinamento de Resistência; DMP = diferença média padronizada Fonte: Autor, 2020. 


\section{Discussão}

As diretrizes atuais da Organização Mundial da Saúde (OMS) sobre atividade física e comportamento sedentário recomendam atividades regulares de fortalecimento muscular para idosos ( $\geq 65$ anos) (Bull et al., 2020).

Evidências de alta confiança demonstram que o equilíbrio e os exercícios funcionais reduzem o índice de quedas nesta população. Evidências de moderada confiança indicam que o risco de lesões pode ser reduzido com treinamento multicomponente (combinações de equilíbrio, força, resistência aeróbia, e agilidade). A diretriz da OMS sugere que os idosos realizem esses exercícios com intensidade moderada ou maior em três ou mais dias por semana, com o objetivo de aumentar a capacidade funcional e prevenir quedas (Bull et al., 2020).

No entanto, por se tratar de recomendações coletivas, para todas as faixas etárias e envolvendo vários tipos de atividades físicas, as diretrizes não fornecem informações mais específicas sobre a população idosa.

No caso específico da COVID-19, e considerando a importância de se manter ativo durante o período de isolamento, analisamos as mais recentes evidências sobre o TR para essa população no momento da pandemia.

A partir das evidências analisadas, observa-se que a maioria dos estudos recomendam o TR domiciliar, isolado ou combinado com outros exercícios físicos (Chaabene et al., 2021; Lakicevic et al., 2020; Machado, Pinto, Brusco, Cadore, \& Radaelli, 2020; Marcos-Pardo, Espeso-García, López-Vivancos, Abelleira Lamela, \& Keogh, 2020; Scartoni et al., 2020; Vitale et al., 2020). O objetivo dos exercícios em domicílio é manter e melhorar a saúde geral de forma segura, promover a independência funcional, e prevenir o potencial agravamento das doenças causadas pela falta de movimento corporal e gasto energético (Lakicevic et al., 2020). O treinamento de resistência domiciliar deve ser promovido como uma modalidade de exercício alternativa, válida e econômica para favorecer a saúde física durante o distanciamento social (Vitale et al., 2020).

Vale destacar que, o único ensaio controlado randomizado incluído na presente revisão apontou que os idosos alocados no grupo experimental (submetidos a 50 minutos de TR, 4 x por semana, durante 24 semanas) melhoraram significativamente a força dos membros inferiores durante o período de isolamento em comparação ao grupo controle, que mantiveram suas atividades habituais. E esses benefícios na força muscular foram provavelmente associados ao programa de treinamento de resistência domiciliar (Vitale et al., 2020). Preservar e melhorar a força de membros inferiores em idosos é um parâmetro essencial para prevenir quedas, fraturas e preservar a independência desse grupo durante a pandemia (Fragala et al. 2019).

Quanto ao tipo de TR (isolado ou combinado), provindos de revisões sistemáticas e metanálises não relataram efeitos estatisticamente significativos para o TR isolado versus treinamento combinado (por exemplo, exercícios combinados de equilíbrio, força e flexibilidade) nas medidas de força e equilíbrio muscular, assim revelando que fazer mais tarefas físicas não é necessário para que ocorram melhoras na função física de idosos (Chaabene et al., 2021).

Sabendo que a maioria das residências carece de equipamentos de treinamento, os estudos avaliados sugerem a realização dos exercícios físicos com o próprio peso corporal (Lakicevic et al., 2020; Scartoni et al., 2020). Estes exercícios, quando realizados de maneira correta, podem ser uma estratégia eficiente para desenvolver a força muscular em idosos, e na maior parte do tempo devem ser baseados em exercícios que se assemelhem as atividades da vida diária (AVDs) como sentar e levantar de uma cadeira, realizar um circuito com degraus segurando objetos simultaneamente, sendo esses esses exercícios capazes de estimular a melhora da propriocepção e consciência corporal, que é muito importante para idosos (Scartoni et al., 2020).

Lakicevic et al. (2020) recomendam que o TR com o peso do próprio corpo deve ser planejado para incluir 8 a 10 exercícios (envolvendo os principais grupos musculares), permitindo 1 a 3 séries por exercício e 10 a 15 repetições por série. 
Marcos-Pardo, Espeso-García, López-Vivancos, Abelleira Lamela, \& Keogh (2020) sugerem a resistência adicional por meio de bandas elásticas, como uma maneira simples de fornecer sobrecarga progressiva no TR. Esse método demonstrou oferecer benefícios semelhantes aos equipamentos de treinamento de resistência tradicionais normalmente encontrados em ginásios, e mostra-se fácil de ser utilizado no ambiente domiciliar, pois não ocupa espaço e é simples de manusear.

Em relação ao volume e intensidade do TR, não houve um consenso entre os estudos avaliados. No entanto, Chaabene et al. (2021), indicam maiores efeitos na força muscular e equilíbrio dos idosos para $>3$ sessões semanais e $\leq 30$ min por sessão, independentemente do tipo de TR. Porém, é necessário monitorar a intensidade durante e após as sessões de treinamento. O uso da escala OMNI-RES validada para idosos (Gearhart, Lagally, Riechman, Andrews, \& Robertson 2009) pode ser uma excelente ferramenta para o controle da intensidade (Scartoni et al., 2020).

Moro e Paoli (2020) apontam para uma combinação do TR e estímulos nutricionais (balanço energético adequado e ingestão de proteínas de alta qualidade) como estratégia eficaz para contrabalançar as perdas no músculo esquelético devido ao desuso durante o período de quarentena.

$\mathrm{O}$ maior achado da presente revisão refere-se à repercussão positiva que o TR exerce sobre função muscular e desempenho funcional em idosos e principais recomendações deste treinamento durante a pandemia da COVID-19, seja como forma de incrementar, manter, prevenir e até reverter os declínios destas variáveis na velhice, independentemente do tipo de intervenção de TR proposta e da metodologia utilizada, assim revelando alta eficiência do TR.

Como limitações desta pesquisa, apontamos a escassez de ensaios clínicos randomizados e estudos com delineamento experimental, estudos com pouco rigor metodológico, o que pode ter sido devido à emergência do tema em estudo.

\section{Conclusão}

As evidências analisadas, apontam para benefícios do TR sobre a função muscular e desempenho funcional em idosos durante a pandemia do COVID-19. O treinamento de resistência domiciliar constitui uma estratégia de intervenção segura, de baixo custo e fácil aplicação, com repercussão positiva para a saúde dessa população.

Os exercícios de fortalecimento com o peso do próprio corpo constituem uma estratégia de intervenção viável e eficiente para a melhoria do condicionamento físico dos idosos durante o isolamento.

Por fim, atentamos que o controle do volume e intensidade é fundamental para uma progressão gradual do treinamento de modo a evitar efeitos nocivos e promover a melhora do sistema imunológico.

\section{REFERÊNCIAS}

American College of Sports Medicine. Exercise is medicine. (2020). Staying physically active during the COVID-19 pandemic. Indianápolis. Disponível em https://www.acsm.org/read-research/newsroom/news-releases/news detail/2020/03/16/staying-physically-active-during-covid-19-pandemic (acesso:11 abr. 2020).

Beyea, S., \& Nicoll, L. H. (1998). Writing an integrative review. AORN Journal, 67(4), 877-880. https://doi.org/10 $.1016 /$ S0001-2092(06)62653-7

Bull, F. C., Al-Ansari, S.S., Biddle, S., Borodulin, K., Buman, P.M., Cardon, G. et al. (2020). World Health Organization 2020 guidelines on physical activity and sedentary behaviour. British Journal of Sports Medicine, 54(24), 1451-1462. Disponível em https://bjsm.bmj.com/content/54/24/1451

Chaabene, H., Prieske, O., Herz, M., Moran, J., Höhne, J., Kliegl, R., Ramirez-Campillo, R., Behm, D. G., Hortobágyi, T., \& Granacher, U. (2021). Home-based exercise programmes improve physical fitness of healthy older adults: A 
PRISMA-compliant systematic review and meta-analysis with relevance for COVID-19. Ageing research reviews, 67, 101265. https://doi.org/10.1016/j.arr.2021.101265

Fragala, M. S., Cadore, E. L., Dorgo, S., Izquierdo, M., Kraemer, W. J., Peterson, M. D., \& Ryan, E. D. (2019). Resistance Training for Older Adults: Position Statement From the National Strength and Conditioning Association. Journal of strength and conditioning research, 33(8), 2019-2052. https://doi.org/10.1519/JSC.00 00000000003230

Gearhart, R. F., Jr, Lagally, K. M., Riechman, S. E., Andrews, R. D., \& Robertson, R. J. (2009). Strength tracking using the OMNI resistance exercise scale in older men and women. Journal of strength and conditioning research, 23(3), 1011-1015. https://doi.org/10.1519/JSC.0b013e3181a2ec41

Gentil, P., Lira, C. A. B., Souza, D., Jimenez, A., Mayo, X., Gryschek, A. L. F. P., Pereira, E. G., Alcaraz, P., Bianco, A., Paoli, A., Papeschi, J., \& Carnevali Junior, L. C. (2020). Resistance training safety during and after the SARSCov-2 outbreak: pratical recommendations. BiomedicalResearch International, 2020 ID 3292916, https://doi. org/10.1155/2020/3292916

Shirvani, H., \& Rostamkhani, F. (2020). Exercise considerations during coronavirus disease (COVID-19) outbreak: A narrative review. Journalof Military Medicine, Tehran, 22(2),161-168.

Lakicevic, N., Moro, T., Paoli, A., Roklicer, R., Trivic, T., Cassar, S., \& Drid, P. (2020). Stay fit, don't quit: Geriatric Exercise Prescription in COVID-19 Pandemic. Agingclinical and experimental research, 32(7), 1209-1210. htt ps://doi.org/10.1007/s40520-020-01588-y

Machado, C., Pinto, R. S., Brusco, C. M., Cadore, E. L., \& Radaelli, R. (2020). COVID-19 pandemic is an urgent time for older people to practice resistance exercise at home. Experimental gerontology, 141, 111101. https://doi.or g/10.1016/j.exger.2020.111101

Marcos-Pardo, P. J., Espeso-García, A., López-Vivancos, A., Abelleira Lamela, T., \& Keogh, J. (2020). COVID-19 and Social Isolation: A Case for Why Home-Based Resistance Training Is Needed to Maintain Musculoskeletal and Psychosocial Health for Older Adults. Journal of aging and physical activity, 29(2), 353-359. https://doi.org/ 10.1123/japa.2020-0131

Moro, T., \& Paoli, A. (2020). When COVID-19 affects muscle: effects of quarantine in older adults. European journal of translational myology, 30(2), 9069. https://doi.org/10.4081/ejtm.2019.9069

Nogueira, C. J., Cortez, A. C. L., Leal, S. M. O., \& Dantas, E. H. M. (2021). Recomendações para a prática de exercício físico em face do COVID-19: uma revisão integrativa. Revista Brasileira de Fisiologia do Exercício, 20(1), 101-124. https://doi.org/10.33233/rbfex.v20i1.4254

Oliveira, W. A., Oliveira-Cardoso, E. A., Silva, J. L., \& Santos, M. A. (2020). Impactos psicológicos e ocupacionais das sucessivas ondas recentes de pandemias em profissionais da saúde: revisão integrativa e lições aprendidas. Estudos de Psicologia, Campinas, (37), e200066. https://doi.org/10.1590/1982-0275202037e200066

Ouzzani, M., Hammady, H., Fedorowicz, Z., \& Elmagarmid, A. (2016). Rayyan: A web and mobile app for systematic reviews. Systematic Reviews, 5(1), 210. https://doi.org/10.1186/s13643-016-0384-4

Page, M. J., McKenzie, J. E., Bossuyt, P. M., Boutron, I., Hoffmann, T. C., Mulrow, C. D., Shamseer, L., Tetzlaff, J. M., Akl, E. A., Brennan, S. E., Chou, R., Glanville, J., Grimshaw, J. M., Hróbjartsson, A., Lalu, M. M., Li, T., Loder, E. W., Mayo-Wilson, E., McDonald, S., McGuinness, L. A., ... Moher, D. (2021). The PRISMA 2020 statement: an updated guideline for reporting systematic reviews. BMJ (Clinical research ed.), 372, $\mathrm{n} 71$. https: //doi.org/10.1136/bmj.n71

Santos, C. M. C, Pimenta, C. A. M., \& Nobre, M. R. C. (2007). The PICO strategy for the research question construction and evidence search. Revista Latino-Americana de Enfermagem,15(3), 508-511. https://doi.org/ 10.1590/S0104-11692007000300023

Scartoni, F. R., Sant'Ana, L. O., Murillo-Rodriguez, E., Yamamoto, T., Imperatori, C., Budde, H., Vianna, J. M., \& Machado, S. (2020). Physical Exercise and Immune System in the Elderly: Implications and Importance in COVID-19 Pandemic Period. Frontiersin psychology, 11, 593903. https://doi.org/10.3389/fpsyg.2020.593903

Vitale, J. A., Bonato, M., Borghi, S., Messina, C., Albano, D., Corbetta, S., Sconfienza, L. M., \& Banfi, G. (2020). HomeBased Resistance Training for Older Subjects during the COVID-19 Outbreak in Italy: Preliminary Results of 
a Six-Months RCT. Internationaljournal of environmental research and public health, 17(24), 9533. https://do i.org/10.3390/ijerph17249533 